\title{
HELIUM DISTRIBUTION FOR THE SUPERCONDUCTING DEVICES IN NSRRC
}

\author{
F. Z. Hsiao, H. C. Li, W. S. Chiou, S. H. Chang, \\ National Synchrotron Radiation Research Center, \\ 101 Hsin-Ann Road, Hsinchu 30076, Taiwan
}

\begin{abstract}
In NSRRC up to five superconducting magnets and one superconducting cavity will be installed in the storage ring. At current stage two superconducting magnets and one superconducting cavity are kept in cold condition by one $450 \mathrm{~W}$ helium cryogenic system. The crucial stable cryogenic condition required for the superconducting cavity is hard to achieve due to the join of superconducting magnets. A second cryogenic system dedicated for the superconducting magnets is planned in the next stage. A switch valve box serves the function for the backup of two cryogenic systems for each other and a 100 meter nitrogen-shielding helium transfer line dedicated for the five superconducting magnets are installed at end of the year 2004. This paper presents the helium distribution design of the two cryogenic systems and the commission result of the recent work.
\end{abstract}

\section{INTRODUCTION}

NSRRC made the decision of using superconducting devices in the storage ring in the year 1999. In next year the project of the helium cryogenic system started and a helium system with a maximum cooling power $450 \mathrm{~W}$ at $4.5 \mathrm{~K}$ and a maximum liquefaction rate $110 \mathrm{l} / \mathrm{hr}$ using liquid nitrogen for precooling had finished all its components construction in Nov. 2002. The installation began in Oct. 2002 and the commission phase ended in Oct. 2003. This helium system is dedicated for the cooling of a $500 \mathrm{MHz}$ superconducting radio frequency (SRF) cavity, where the cavity was installed in the storage ring and finished its commission in Jan. 2005. The helium system includes one $315 \mathrm{~kW}$ compressor, one 45 $\mathrm{kW}$ recovery compressor, one $10 \mathrm{~kW}$ refrigerator, one 2000 liter dewar, and two $100 \mathrm{~m} 3$ gas helium buffer tanks. Inside the cold box two expansion turbines connected in series provide the major cooling power for helium gas stream. Two $80 \mathrm{~K}$ adsorbers and one $20 \mathrm{~K}$ adsorber are installed in the cold box. The switch and regeneration of the two $80 \mathrm{~K}$ adsorbers are fully automatic and performed without interrupting the cold box operation. There are two analyzers monitoring the impurity level of the helium stream. One analyzer monitors the oxygen and humidity level at the cold box side; the other one monitors the oil aerosol and nitrogen level at the compressor side. The designed thermal dynamic state and preliminary test result of this helium system can be found in references [1,2].

In addition to the SRF cavity, two superconducting magnets are in operation and cooled by the helium cryogenic system for SRF cavity. There are scheduled another three superconducting magnets to be installed in the storage ring. The required total cooling power exceeds the capacity of the current helium system and the crucial stable cryogenic condition from the SRF cavity is hard to achieve due to the join of superconducting magnets. A second helium cryogenic system dedicated for the superconducting magnets will be installed in the year 2006. This paper presents the helium distribution design of the two cryogenic systems and the commission result of the recent work.

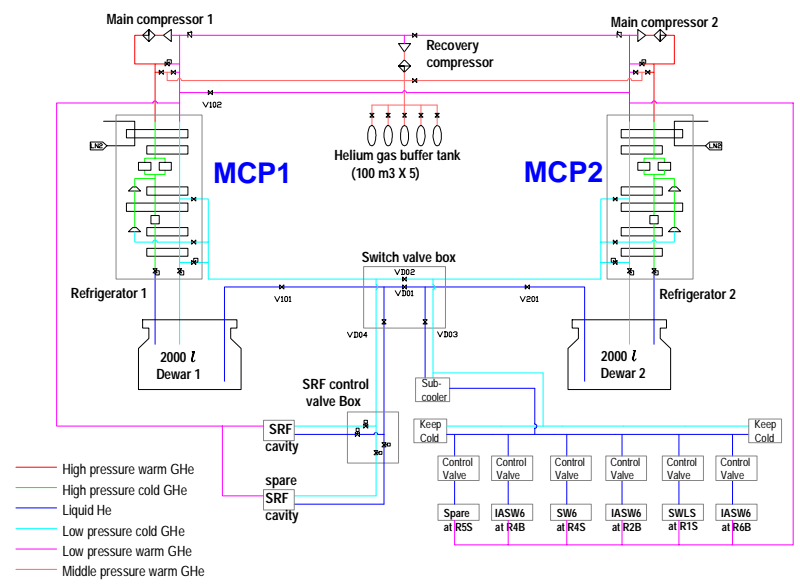

Figure 1: Flow chart of the helium cryogenic system.

\section{CONFIGURATION}

One goal of the helium distribution design is separating the helium stream for the SRF cavity from that for the superconducting magnets, the other goal is providing the backup function for the two helium cryogenic systems. Figure 1 shows the flow chart for the two helium systems and all the superconducting devices [3]. Liquid helium produced from two refrigerators are accumulated in their two storage dewars. The stored liquid helium in the two dewars are first transferred into a switch valve box and then to the superconducting devices. The valve box serves the switch function for the backup of the two cryogenic systems for each other. All the cryogenic valves inside the switch valve box are manual operated valves. The switch valve box has two outlets and provides two helium streams to the SRF cavity and the five magnets respectively. One outlet connects a nitrogen-shielding helium transfer line with length 100 meters for the five superconducting magnets; the other one connects a transfer line with length 3 meters for the SRF cavity. A valve box with control valves inside are installed in between the switch valve box and the SRF cavity. The control valves regulate the pressure and the 
level of the cavity cryostat. Inside all multichannel transfer lines there are a liquid nitrogen line of diameter 0.5 -inch, a liquid helium line of diameter 0.75 -inch, and a cold helium line of diameter 1.25 -inch. It is common vacuum for the insulation vacuum of one multichannel transfer line. The switch valve box has its individual vacuum that is separated from the vacuums of the two inlet multichannel lines and the vacuums of the two outlet multichannel lines. The five superconducting magnets are located below the $100 \mathrm{~m}$ multichannel line, where at each supply port two control-valves regulate the level of liquid nitrogen and liquid helium of the magnet cryostat. After these two control-valves a 0.5 -inch flexible helium supply line and a 0.5 -inch nitrogen supply line are connected to the magnet cryostat. Two keep-colds for helium and two gas-vents for nitrogen are installed at both ends of the 100-m multichannel line. The cold helium gases from the keep-colds are returned to the refrigerator, the cold nitrogen gas from the gas-vents are warmed up by passive warmers and then vented to air. To assure a single liquid helium phase is transferred into this long multichannel line, a subcooler is installed in between the switch valve box and the long multichannel line to condense the vaporized helium gas caused from the transfer heat loss and the heat loss from the switch valve box. Each keepcold or subcooler has its level meter and controller to regulate the level of liquid helium in its buffer dewar.

In normal operation the helium stream for the SRF cavity is separated from the stream for the superconducting magnets. The separated helium streams minimize the interaction of cryostat conditions for the SRF cavity and the superconducting magnets. The pressure fluctuation is desired below $\pm 0.058 \mathrm{psi}$ ( \pm 4 mbar) in the dewar and $\pm 0.0435 \mathrm{psi}( \pm 3$ mbar) in cavity cryostat for a long run. The backup function of the helium distribution is important. Maintenance or safety inspection of the major components of the helium system takes several days and this action will open the system to air, thus two weeks is required for the helium system recovery. It is difficult to keep the cavity at low temperature over one day using the inventory liquid helium in the dewar, since the heat load from the SRF cavity without operation is $30 \mathrm{~W}$ and the heat loss for helium transfer is $30 \mathrm{~W}$. Before stopping the helium system, it needs to warm up the SRF cavity and the superconducting magnets. The time required to warm up the SRF cavity and the superconducting magnets to room temperature is more than 12 days and it takes one week to cool down the SRF cavity from room temperature to 4.5 K. A shutdown of the storage ring with a period five weeks is thus required for the maintenance of the cryogenic system. The backup of the cryogenic system makes it possible to schedule the maintenance or safety inspection of the two helium cryogenic systems without causing the shutdown of the SRF cavity and the storage ring. However, the cooling capacity of a helium system can not serve the cooling requirements from all the superconducting devices in the storage ring. Some of the superconducting magnets and their corresponding beamlines may be forced to shutdown during the maintenance period of the helium system.

\section{RESULT}

The switch valve box and the long multichannel line were installed at end of the year 2004. The helium lines are inner polished piping. We controlled the dew point below $223 \mathrm{~K}$ and the particle number below the class- 100 standard before connecting the helium piping to the helium system. Figure 2 shows the pumping down curves of the insulation vacuum of both the long multichannel line and the switch valve box. Two $250 \mathrm{l} / \mathrm{s}$ turbo pumps were connected near both ends of the long multichannel line and one $250 \mathrm{l} / \mathrm{s}$ turbo pump was used for the switch valve box. Because of the limited conductance of the $40 \mathrm{KF}$ pumping port and the high outgassing rate, it took ten days to pump down the insulation vacuum of the long multichannel line to a pressure $2.5 \mathrm{e}-4$ torr. After one week pumping the insulation vacuum of the switch valve box reached a pressure $2.15 \mathrm{e}-5$ torr. The leak rate of all the transfer lines to insulation vacuum at warm condition is below 2e-8 mbar-1/sec for the long multichannel line and $3 \mathrm{e}-10 \mathrm{mbar}-1 / \mathrm{sec}$ for the switch valve box. The leak rate at cold condition is $2.8 \mathrm{e}-9 \mathrm{mbar}-1 / \mathrm{sec}$ for the long multichannel line and $9.9 \mathrm{e}-10 \mathrm{mbar}-1 / \mathrm{sec}$ for the switch valve box. As the pressure down to the acceptable vacuum the insulation vacuum of all transfer lines and the switch valve box are maintained statically without permanent connection to a pump. The 24-hour vacuum retention test indicated the pressure rising from $1.52 \mathrm{e}-4$ torr to $1.19 \mathrm{e}-3$ torr for the switch valve box, and the pressure rising from $2.36 \mathrm{e}-3$ torr to $4.49 \mathrm{e}-3$ torr for the long multichannel line.

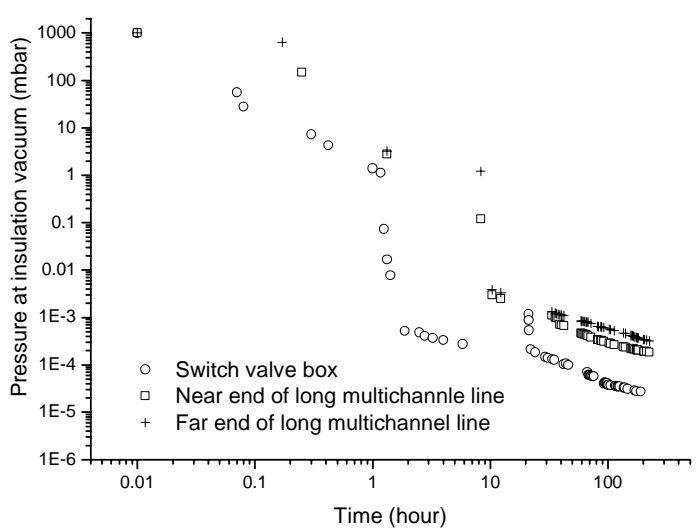

Figure 2: Pumping down curves of the insulation vacuum.

It took 5 hours to cool down the long multichannel line from room temperature to $4.2 \mathrm{~K}$ and the time required to naturally warm up this line to room temperature is 5 days. The total heat load of the switch valve box and the long multichannel line, as Fig. 3 shows, is $105 \mathrm{~W}$. This value is obtained from the difference of heater power in the dewar with or without liquid helium flowing through the long multichannel line. 


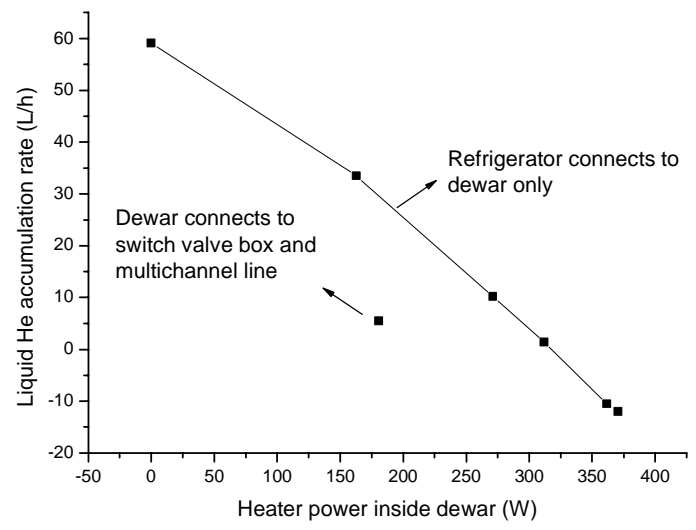

Figure 3: Heat load measurement.

A superconducting magnet is connected at the port near the subcooler; a magnet is located near the far end of the long multichannel line. The helium pressures of the magnet cryostats are $15.12 \mathrm{psig}(1.042 \mathrm{barg})$ with a fluctuation $\pm 0.012 \mathrm{psi}( \pm 0.83 \mathrm{mbar})$ and $15.31 \mathrm{psig}$ (1.056 barg) with a fluctuation \pm 0.05 psi $( \pm 3.45$ mbar $)$ respectively. Both magnet cryostats are operated at liquefaction mode without the cold helium gas returning back to the refrigerator. Figure 4 shows helium levels of the keep-colds and the subcooler of the long multichannel line, pressures of the helium supply and the cold return gas is indicated in Fig.5. The keep-cold at far end of the multichannel line can not keep its helium level as the magnet near the subcooler fills liquid helium. Both pressures of the helium supply and the cold return gas are disturbed by the filling actions of the two magnets, where the magnet near the subcooler is filled at every 25 minutes and the other one is filled at every 7 hours. The pressure fluctuation affects operation of the SRF cavity as helium for the two superconducting magnets and the SRF cavity are from the same cryogenic system.

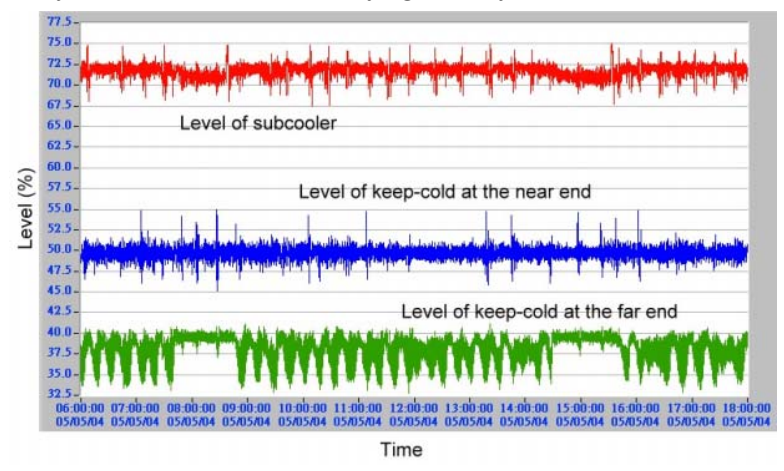

Figure 4: Levels in keep-colds and subcooler.

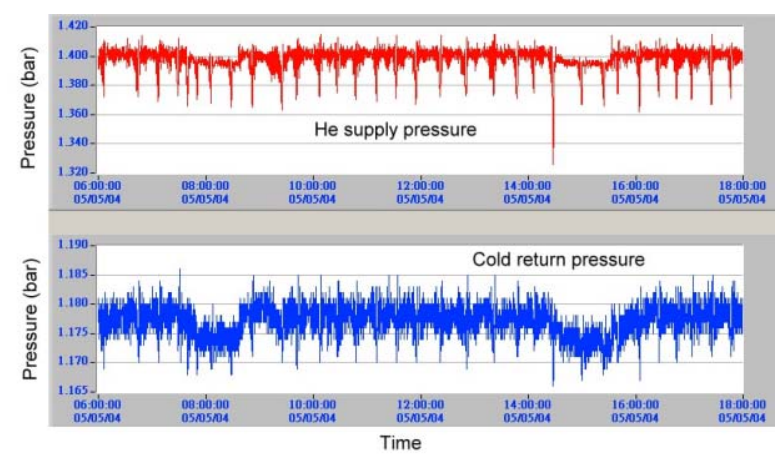

Figure 5: Pressure of the helium lines.

\section{SUMMARY}

The helium distribution for the superconducting devices in NSRRC had finished its framework and is successful in operation. The next stage will be the integration of the second cryogenic system and then the additional three superconducting magnets.

\section{ACKNOWLEDGEMENT}

The authors wish to thank Mr. Frank Ypma, Mr. Marco Ilonka, and Mr. Marcel Keezer from DeMaCo for their effort during the construction of the helium distribution system.

\section{REFERENCES}

[1] F. Z. Hsiao, et al., "The Liquid Helium Cryogenic System for the Superconducting Cavity in SRRC", PAC'01, Chicago, Illinois, June 2001, p. 1604-1606.

[2] F. Z. Hsiao, et al., "The Pilot-Runs of the Helium Cryogenic System for the TLS Superconducting Cavity", PAC'03, Oregon, Portland, May 2003, p. 2402-2404.

[3] "Technical Specification: Helium Transfer System for the Six superconducting Magnets at NSRRC," NSRRC Technical Report, Oct. 15, 2003. 